\title{
Marcel Jousse
}

Professeur d'Anthropologie linguistique à l'École d'Anthropologie

(1886-1961)

\section{"Les lois psycho-physiologiques du Style oral vivant et leur utilisation philologique." \\ Revue d'ethnographie, no 23, 1931.}

\section{Un document produit en version numérique par Mme Marcelle Bergeron, bénévole Professeure à la retraite de l'École Dominique-Racine de Chicoutimi, Québec Courriel: mailto:mabergeron@videotron.ca \\ Page web}

Dans le cadre de la collection: "Les classiques des sciences sociales" Site web: http://classiques.uqac.ca/

Une collection développée en collaboration avec la Bibliothèque Paul-Émile-Boulet de l'Université du Québec à Chicoutimi Site web: http://bibliotheque.uqac.cal 


\section{Politique d'utilisation de la bibliothèque des Classiques}

Toute reproduction et rediffusion de nos fichiers est interdite, même avec la mention de leur provenance, sans l'autorisation formelle, écrite, du fondateur des Classiques des sciences sociales, JeanMarie Tremblay, sociologue.

Les fichiers des Classiques des sciences sociales ne peuvent sans autorisation formelle:

- être hébergés (en fichier ou page web, en totalité ou en partie) sur un serveur autre que celui des Classiques.

- servir de base de travail à un autre fichier modifié ensuite par tout autre moyen (couleur, police, mise en page, extraits, support, etc...),

Les fichiers (.html, .doc, .pdf, .rtf, .jpg, .gif) disponibles sur le site Les Classiques des sciences sociales sont la propriété des Classiques des sciences sociales, un organisme à but non lucratif composé exclusivement de bénévoles.

Ils sont disponibles pour une utilisation intellectuelle et personnelle et, en aucun cas, commerciale. Toute utilisation à des fins commerciales des fichiers sur ce site est strictement interdite et toute rediffusion est également strictement interdite.

\section{L'accès à notre travail est libre et gratuit à tous les utilisateurs. C'est notre mission.}

Jean-Marie Tremblay, sociologue

Fondateur et Président-directeur général, LES CLASSIQUES DES SCIENCES SOCIALES. 
Un document produit en version numérique par Mme Marcelle Bergeron, bénévole, professeure à la retraite de l'École Dominique-Racine de Chicoutimi, Québec. Courriel : mabergeron@videotron.ca

Marcel Jousse.

"Les lois psycho-physiologiques du Style oral vivant et leur utilisation philologique."

Une communication publié dans la Revue d'ethnographie, $46^{e}$ année, no 23, 1931. $18 \mathrm{pp}$. Communication faite au $15^{e}$ congrès international d'anthropologie tenu à Paris à la Faculté de médecine du 20 au 27 septembre 1931, $5^{e}$ section : ethnographie.

Le directeur pédagogique de l'Institut européen de mimopédagogie, M. Yves Beauperin, nous a donné sa permission, le 25 juin 2003, de duffuser cette œuvre dans Les Classiques des sciences sociales.

Police de caractères utilisée:

Pour le texte: Comic Sans, 12 points.

Pour les notes de bas de page: Comic Sans, 10 points.

Édition électronique réalisée avec le traitement de textes Microsoft Word 2008 pour Macintosh.

Mise en page sur papier format : LETTRE US, $8.5^{\prime \prime} \times 11^{\prime \prime}$ )

Édition réalisée le 22 février 2004 et complétée le 6 décembre 2010 à Chicoutimi, Québec. 
à M. le Dr Joseph Morlaâs 


\title{
COMMUNICATION
}

FAITE

\section{AU XV CONGRÈS INTERNATIONAL D'ANTHROPOLOGIE}

\author{
TENU À PARIS \\ À LA FACULTÉ DE MÉDECINE \\ DU 20 AU 27 SEPTEMBRE 1931
}

$5^{e}$ Section : Ethnographie. 
[1]

\title{
LINGUISTIQUE
}

\author{
Les Lois psycho-physiologiques, \\ du Style oral vivant \\ et leur utilisation philologique
}

\section{MARCEL JOUSSE}

Les pages qui suivent voudraient être un essai de collaboration plus étroite, sur des sujets partiellement communs, entre phonéticiens expérimentaux, psychologues linguistes et ethnographes ${ }^{1}$.

Il y a une vingtaine d'années, l'orientation de nos études scientifiques nous amenait à nous poser ce problème de psychologie expÉrimentale et ethnique: "Comment I'homme, placé au milieu des innombrables actions de l'univers, s'y prend-il pour conserver en lui le souvenir de ces actions et pour le transmettre fidèlement, de génération en génération, à ses descendants? » Au fond, il ne s'agissait de rien de moins que d'étudier, objectivement, l'élaboration des outils psycho-physiologiques qui servent au grand fait humain de la Tradition vivante.

Sur la nécessité de cette collaboration, voir l'excellent paragraphe de B. NIKITINE dans /'Ethnographie, Nouvelle Série, $n^{\circ s} 19$ et 20 (1929), p. 112. Voir aussi : Dr Joseph MORLAAS: Contribution à l'étude de l'Apraxie, Paris, 1928. Dr Georges Dumas: Nouveau Traité de Psychologie, t. III., chapitre sur le Langage, par le Dr A. OMBREDANE (préalablement publié dans la Revue philosophique, nos 3 et 4 (1931), pp. 217 et suiv.). 
Lentement et méthodiquement, soucieux avant tout de ne rien inventer, nous nous sommes mis alors à recueillir des faits. Le plus grand nombre possible de milieux ethniques, d'un bout du monde à l'autre, ont été l'objet de notre enquête. Mais il va de soi que notre attention s'est portée, de préférence, sur les groupements humains qui sont encore à l'abri de l'imprimerie. Il serait, en effet, singulièrement antiscientifique de prétendre juger des possibilités [2] de la mémoire humaine d'après des sujets qui s'ingénient à s'en passer, sûrs qu'ils sont de tout retrouver, en cas de besoin, dans le livre approprié ou dans le dictionnaire encyclopédique.

Et cependant, que de questions scientifiques très graves ont été psychologiquement faussées -- et même rendues insolubles -- par l'attribution intempestive de notre grandissante «amnésie» à des individus de milieux ethniques profondément différents du nôtre sur ce point.

$\grave{A}$ nos observations ethnographiques personnelles, nous avons joint les renseignements recueillis au cours de nombreuses conversations avec des explorateurs et des missionnaires. Les relations écrites par ces grands observateurs du «Laboratoire ethnique», depuis trois siècles surtout, nous ont été également précieuses pour orienter nos enquêtes.

Le cinématographe, le phonographe et, les merveilleux appareils enregistreurs du génial et regretté professeur du Collège de France, l'abbé J.-P. Rousselot, sont venus ajouter leur précision objective et expérimentale à ces investigations, complexes comme tout ce qui touche à la vie.

Après dix-huit ans de ces laborieuses et passionnantes recherches, nous avons publié, dans les Archives de Philosophie, une "esquisse 》 de notre méthode expérimentale et quelques-uns des résultats auxquels nous étions parvenu. Le mémoire a paru au début de 1925 sous ce titre : Études de Psychologie linguistique: le Style oral rythmique et mnémotechnique chez les Verbo-moteurs (Paris, Beauchesne). Chaque chapitre y résume chacun de nos futurs ouvrages que les matériaux vivants ont, pour ainsi dire, composés d'eux-mêmes, en s'ordonnant selon la logique des faits. 
Pour une explication plus ample et plus psychologique des faits rapidement mentionnés dans les pages qui vont suivre, nous nous permettons donc de renvoyer les lecteurs à ce mémoire précédemment publié.

La complexité de la matière est telle qu'à force de « résumer des résumés », on arrive malgré soi à donner une idée fausse de la réalité.

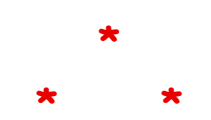

Toute étude sur la psychologie du langage, disions-nous dans les premiers chapitres de notre travail, doit commencer par un examen approfondi de la proposition vivante, du geste propositionnel. Cet examen expérimental des procédés expressifs de l'homme est vraiment trop compliqué pour que nous songions, ici, à en donner même une simple analyse. Il nous faudrait, en effet, partir d'une étude cinématographique des admirables langages de [3] gestes ${ }^{2}$, cause première et encore inexplorée des écritures dites, «hiéroglyphiques» et qu'il serait plus juste de nommer «mimographiques». Nous aurions ensuite à passer par des langues intermédiaires, mais toujours concrètes, comme le chinois. Enfin, par delà les syllogismes algébrisants d'un Aristote, nous aboutirions aux formules relationnelles de la haute analyse mathématique d'un Henri Poincaré et d'un Einstein. «Du Concrétisme à l'Algébrisme » : c'est un beau sujet pour une vie d'études, mais un peu vaste pour la présente esquisse.

Contentons-nous donc de dire que ces recherches nous ont permis de montrer l'origine psycho-physiologique du phénomène linguistique connu, depuis Lowth, sous le nom de parallélisme des propositions. Mais ce que, à son époque, Lowth ne pouvait pas soupçonner, c'est l'importance

Marcel Jousse, Méthodologie de la Psychologie du Geste.I, le Style manuel. (Revue des Cours et Conférences, 15 mai 1931, pp. 201-218.) 
psychologique énorme de ce phénomène ${ }^{3}$. Sans exagération aucune, on peut affirmer qu'il joue, dans le monde de la pensée et de la mémoire humaine, un rôle aussi central que la gravitation dans l'univers physique.

En effet, de par les lois profondes du «composé humain» fait de chair et d'esprit, chaque proposition improvisée a une curieuse tendance à déclencher, dans le système phonateur du sujet parlant, une ou deux autres propositions parallèlement construites et de sens analogue ou antithétique ${ }^{4}$.

Par exemple, recueillons au phonographe les délicieuses improvisations d'un compositeur oral de l'Emyrne, région située au centre de Madagascar. Après une analyse linguistique des propositions enregistrées sur les disques, nous serons frappés par un parallélisme nettement accusé. Même une traduction, toujours déformante, nous permet d'en sentir encore les rudiments:

L'argent est la corne du riche,

la bêche est la corne du pauvre.

Vous êtes au milieu de mille citronniers,

je suis au milieu de mille foules.

Je ne suis pas le chat sauvage insouciant, mais le chat qui obéit à une loi. ${ }^{5}$

Nous pouvons promener notre phonographe enregistreur, d'un bout du monde à l'autre, dans tous les milieux de «style oral », [4] c'est-à-dire dans tous les groupements ethniques qui ne connaissent pas l'écriture ou

3 Voir Louis MARIN, Un exemple des rapports entre les études ethniques et anthropologiques: les systèmes de versification. (Institut international d'Anthropologie. III session, Amsterdam, 20-29 septembre 1927. Paris, 1928, pp. 493-499.)

4 J. Van GINNEKEN, Principes de Linguistique psychologique (Paris-Leipzig, 1907), pp. 528-529.

5 J. PAULHAN, Les hain-teny merinas (Paris, 1913), p. 355. 
qui ne s'en servent pas pour composer. Avec des variations nombreuses, dues à la souplesse de la vie, au mécanisme des diverses langues et aussi, empressons-nous de le dire, à la volonté libre de l'homme capable de briser bien des automatismes, le résultat sera le même ${ }^{6}$.

Un phénomène connexe, de nature également. psycho-physiologique, attirera encore notre attention. D'ailleurs, le cinématographe enregistreur nous le conservera précieusement et nous aidera à l'étudier à loisir.

Nous voulons parler d'un balancement caractéristique qui fait osciller tout le corps du récitateur et accompagne généralement le débit de chaque proposition ${ }^{7}$. Certains groupes ethniques ont comparé ce balancement au dandinement du chameau chargé d'un fardeau, au rengorgement de la colombe qui roucoule, etc.

Chacune des propositions parallèles ou, comme nous allons désormais les appeler, chacun de ces balancements se module sur une mélodie simple et assez monotone. Les membres mélodiques de cette psalmodie se balancent, eux aussi, naturellement, selon le parallélisme des propositions qu'ils animent. Les deux ou trois émissions vocales, sémantiquement et mélodiquement parallèles, forment ainsi un tout complexe, une sorte de schéma vivant, binaire ou ternaire, que nous avons nommé schème rythmique.

Pour être clair et concret, voici un exemple de schème rythmique binaire, c'est-à-dire composé de deux balancements :

Ils ne conseillent pas d'abord, mais ils raillent plus tard.

Voir, par exemple, Elias LONROT, le Kalevala, trad. par J.-L. PERRET (Paris; 1931).

J.-A. JAUSSEN, Coutumes palestiniennes: I. Napelouse et son district (Paris, 1927), p. 81. 
Voici un exemple de schème rythmique ternaire, c'est-à-dire composé de trois balancements :

Nous ne les chassons pas devant nous, comme les moutons, mais ils viennent en suivant, comme les chiens, ils se balancent par derrière, comme une queue de mouton. ${ }^{8}$

Pour bien montrer que les deux ou trois balancements d'un schème rythmique forment une unité de récitation, une unité vivante, mélodique et équilibrée -- et non pas deux ou trois fragments erratiques -- nous avons adopté la graphie ci-dessus, avec [5] des lignes en retrait. Mais ce n'est qu' une grossière indication. Elle ne remplacera jamais la récitation réelle, ni même son mélodieux écho phonographique.

Nous voulons signaler tout de suite à nos lecteurs l'importance exceptionnelle de cette question de la mélodie et du rythme des propositions orales. En effet, nous ne dirons jamais assez quel secours les ethnographes apporteront à la science psychologique et rythmique de demain en recueillant, sur des disques phonographiques et mieux encore sur les appareils phonétiques Rousselot, le plus possible d'éléments vivants des propositions ethniques spontanées ${ }^{9}$.

Chaque balancement de chacun des schèmes rythmiques improvisés peut être ainsi analysé, objectivement et à l'aide du microscope, dans tous ses éléments phonétiques vivants et si effroyablement complexes: rythme d'intensité, rythme de durée, rythme de timbre, rythme de hauteur, allitération des consonnes, allitération des voyelles, nombre exact de syllabes réellement prononcées, tempo moyen du débit, etc., etc. ${ }^{10}$.

J. PAULHAN, les hain-teny merinas (Paris, 1913), p. 387.

Abbé ROUSSELOT, Principes de Phonétique expérimentales (2 vol., Paris, 1924).

$G$. LOTE, Études sur le vers français : l'alexandrin d'après la Phonétique expérimentale $^{2}$ (2 vol., Paris, 1924). 
Et qu'on veuille bien le remarquer: chacun de ces innombrables éléments est présent et agissant dans la mystérieuse genèse de toute proposition orale et dans sa conservation mnémonique. $\grave{A}$ des degrés divers, d'ailleurs, et que seuls peuvent révéler et doser, avec une mathématique exactitude, les graphiques des appareils enregistreurs de la phonétique expérimentale.

En effet, depuis les belles découvertes de l'abbé Rousselot, on ne peut plus guère étudier à son bureau, uniquement avec une plume et du papier, comme jadis, ces jeux terriblement complexes de l'énergie vivante et que I'on nommait, par une simplification excessive, le rythme. Ce rythme n'était bien souvent, en effet, et -- psychologiquement -- ne pouvait guère être que telle ou telle idée préconçue d'un linguiste allemand, anglais, français, italien, etc., infligeant inconsciemment ses habitudes linguistiques ethniques à de mortes et passives lignes d'écriture.

$C^{\prime}$ 'est ainsi que, dans une thèse de doctorat en Sorbonne ${ }^{11}$ un métricien «syllabiste » français voulait, coûte que coûte, réduire à huit syllabes chacun des souples balancements des improvisateurs basques. Or, ces balancements varient, ad libitum, entre [6] cinq et quinze syllabes environ au cours d'une même improvisation. Les improvisateurs basques ont d'ailleurs protesté contre cette docte vivisection ${ }^{12}$.

De même, certains métriciens allemands, accoutumés à la régularité des ictus intensifs, prétendaient, au nom du rythme, que nous rythmions ainsi notre Racine avec leur accent d'intensité :

Oui, c'est Agamemnon, c'est ton roi qui t'éveille

11 A. LÉON, Une Pastorale basque : Hélène de Constantine (Paris, 1908), p. 511.

12 HÉRELLE, Études sur le théâtre basque : les Pastorales à sujets tragiques considérées littérairement (Paris, 1926), p. 62. 
Nous aurions alors trois ictus, bien réguliers, dans chaque membre récité ${ }^{13}$. Naturellement, nous autres Français, nous sourions de ce rythme qui n'a qu'un défaut : celui de ne point exister sur nos lèvres.

Encore est-il que pour sourire ou protester -- ce qui est bien un peu la même chose -- il faut être vivant! Or, c'est cette vie, doucement mais objectivement rectificatrice, que n'ont malheureusement plus les textes écrits et surtout les textes écrits des langues lointaines ou disparues.

Aussi, les palestinisants, informés des innombrables essais de rythmique hébraïque, par exemple, savent quelles opérations chirurgicales certains métriciens ont fait subir -- metri gratiâ -- aux traditionnelles mais dociles propositions des psalmistes et des prophètes d'Israël.

Il suffit d'ouvrir au hasard et de lire quelques-unes de ces impitoyables « rythmisations » pour entendre, souvent plusieurs fois par page, le son lugubre du couperet ou de l'extenseur automatique: « retrancher ce mot pour le rythme! ajouter un mot pour le rythme!» (Ne faudrait-il pas parfois traduire: « pour mon rythme »?).

En etfet, avant d'oser, au nom du rythme et du rythme seul, toucher même légèrement à de pareils textes traditionnels, la première chose à faire, semble-t-il, était de commencer par analyser scientifiquement la nature psycho-physiologique de ce rythme mystérieux et -- dans l'occurrence -- dévastateur. Il restait ensuite à mener de méticuleuses enquêtes ethniques pour étudier le plus grand nombre possible de rythmiques vivantes et spontanées. Or, dans le cas présent, le psychologue et le phonéticien expérimental constatent, avec surprise, que ce tte préalable investigation a été étrangement négligée. Et pourtant, il suffisait d'étendre cette enquête à une centaine de milieux ethniques indépendants [7] pour voir s'en dégager des lois rythmiques vivantes et souples, dans lesquelles viennent s'insérer, d'elles-mêmes et sans mutilation, les antiques propositions des psalmistes et des prophètes hébreux.

13 G. LOTE, Études sur le vers français : I'alexandrin d'après la Phonétique expérimentale ${ }^{2}$ (Paris, 1919), t. II, p. 467. 
Par là, nous voyons combien, en matières scientifiques, il est dangereux d'imaginer au lieu d'observer. Le manque d'informations objectives se change vite en pseudo-problèmes et en difficultés apparemment déconcertantes. Or, au fur et à mesure de la spécialisation scientifique, fatalement imposée aux chercheurs modernes, la complexité des questions va croître de jour en jour. Aussi, ne pourront-elles plus être résolues que par l'intime et constante -- et fraternelle -- collaboration des diverses disciplines, rationnelles et expérimentales.

Dans la branche scientifique très neuve qui nous occupe en ce moment, la plupart des ethnographes et des linguistes se sont trouvés en contact avec des documents vivants d'une importance unique et maintenant disparus à jamais. Faute de moyens matériels et faute d'initiation aux méthodes expérimentales, ils ne nous ont guère donné, jusqu'ici, que de vagues transcriptions graphiques des paroles mélodieusement et rythmiquement balancées. Chaque linguiste, allemand, anglais, français, italien, etc., les transpose alors et les rythme sur ses lèvres selon sa manière ethnique et toujours déformante.

Soyons heureux d'avoir vu récemment s'ouvrir aux phonéticiens, aux linguistes, aux rythmiciens, ce que tant de techniciens rêvaient depuis si longtemps: les archives de la parole et du geste. Là, d'innombrables disques, enregistrés par l'admirable et intelligente équipe des ethnographes et des missionnaires, nous rediront, dans leur mélodie vivante, les récitations ethniques dont les revues ethnographiques nous apportaient déjà les graphies précieuses, mais décharnées.

Car il faut le rappeler sans cesse, puisque notre effrayant « graphisme » moderne nous le fait trop oublier et ne nous permet plus de résoudre les problèmes linguistiques complexes et vivants: dans le langage humain réel, il n'y a pas d'un côté les paroles, d'un autre côté les mélodies, d'un autre côté encore les rythmes, etc. Non, mais tout cela 
joue et se compénètre intimement pour déclencher et diriger dans l'organisme les subtils balancements parallèles ${ }^{14}$.

Ce parallélisme vivant et agissant, à la fois physiologique, sémantique, mélodique et rythmique, finit par «stéréotyper», [8] pour ainsi dire, la musculature d'un certain nombre de proverbes-types dans chaque milieu de style oral.

Prenons un de ces milieux ethniques dont les quatre ou cinq cents proverbes-types sont fixés pour toujours et transmis sans modification par la tradition orale ${ }^{15}$. L'invention personnelle des improvisateurs consistera, dès lors, à prendre plus ou moins consciemment pour modèles ces quatre ou cinq cents schèmes rythmiques-types; puis à ajuster sur eux d'autres schèmes rythmiques de forme pareille, ayant même structure, même nombre de mots, mêmes rythmes et, dans la mesure du possible, même sens.

Soit, par exemple, dans le milieu de style oral merina ${ }^{16}$, I'un quelconque de ces quatre ou cinq cents proverbes-types

Ce n'est pas la pluie qui vient petite, petite, mais c'est notre conversation à tous deux qui est petite, petite.

Nous entendrons, sur les lèvres des improvisateurs, des schèmes rythmiques d'imitation, pareils à celui-ci :

14 Sur cette intime compénétration, voir la thèse de Stoyan DJOUDJEFF: Rythme et Mesure dans la Musique populaire bulgare, Paris, 1931. -- Voir aussi ; Th. B. van LELYVELD, la Danse dans le Théâtre javanais, Paris, 1931.

15 J. PAULHAN, I'Expérience du Proverbe: Cahiers Commerce, 1925, Cahier V., p. 28.

16 J. PAULHAN, les hain-teny merinas, pp. 52-58. 
Ce ne sont pas les rizières dont le riz est petit, petit, mais c'est notre affection à tous deux qui est petite, petite.

Les schèmes rythmiques improvisés au cours des compositions orales sont donc modelés sur ces schèmes rythmiques-types. Ils les reproduisent à des centaines d'exemplaires nouveaux, ils les développent ou les abrègent parfois, ils les entourent de propositions différemment rythmées qui, cependant, sont calquées, elles aussi, sur d'autres proverbes-types. On a ainsi une idée très approchée de ce qu'est le style oral rythmique dans un milieu d'improvisateurs.

Comme nous l'avons vu jusqu'ici, l'action psycho-physiologique du parallélisme s'exerce normalement et habituellement de balancement à balancement à l'intérieur du schème rythmique.

Pourtant, il n'est pas rare de surprendre son influence sur les schèmes rythmiques eux-mêmes qui deviennent ainsi parallèles entre eux, comme le sont les binaires et ternaires suivants:

Cette fumée, vers l'Ouest,

n'est pas de la fumée, mais une coquetterie.

Ce riz que l'on pile,vers I'Est,

$n^{\prime}$ est pas du riz que l'on pile, mais un caprice ${ }^{17}$

J. PAULHAN, les hain-teny merinas, p. 19. 
[9]

-- Les tubercules précèdent l'ambiaty:

suis-je l'amie qui n'est pas aimée

que vous me réveilliez quand le ciel à l'horizon est sombre?

-- Les tubercules précèdent l'ambiaty:

vous n'êtes pas l'amie qui n'est pas aimée

et je vous réveillerais quand le ciel à l'horizon est sombre? ${ }^{18}$

Le parallélisme est même tellement actif dans toutes les fibres humaines qu'il tend à balancer parallèlement jusqu'à des groupes entiers de propositions, parfois déjà parallèles entre elles deux à deux ou trois à trois. C'est là surtout que se fait sentir le rôle profond et décisif de la mélodie, de l'air sur lequel se modulent ces propositions parallèlement groupées.

Ces groupements instinctifs d'un nombre égal de schèmes rythmiques forment ainsi de petits récitatifs parallèles que nous retrouvons sur les lèvres des improvisateurs du monde entier. En voici un exemple ${ }^{19}$, pris dans le milieu de style oral merina: 


\section{Récitatif 1}

1. Puis-je entrer, Rasao-la-précieuse?

-- Qui demande?

2. -- C'est moi, Audriakoto-de-la-vie, petit qui revêts le lamba rouge à la bordure de pourpre.

3. J'ai un salaka de soie, mes dents sont déboisées.

4. Je monte le Beau-qui-regarde-le-ciel, je fais galoper le Grand-souffle-de-vie.

5. Je lave mes pieds avec du lait, je lave ma bouche avec du miel.

6. J'apporte l'argent au poids exact, j'apporte les morceaux de piastre.

7. -- Si vous apportez l'argent au poids exact, si vous apportez les morceaux de piastre,

8. Notre veau est mal attaché, notre porte est bien fermée, père et mère dorment ici. 


\section{Récitatif 2}

1. Puis-je entrer, Rasoa-la-précieuse?

-- Qui demande?

2. -- C'est moi, Andriakoto-de-la-vie, petit qui revêts le lamba rouge à la bordure de pourpre.

3. J'ai un salaka de soie, mes dents sont déboisées.

4. Je monte le Beau-qui-regarde-le-ciel, je fais galoper le Grand-souffle-de-Vie.

5. Je lave mes pieds avec du lait, je lave ma bouche avec du miel.

6. J'apporte la viande bien grasse, j'apporte les petites boules de graisse.

7. -- Si vous apportez la viande bien grasse, si vous apportez les petites boules de graisse,

8. Notre veau est bien attaché, notre maison est mal fermée, père et mère ne dorment pas ici. 
Ces curieux et universels récitatifs parallèles peuvent être ainsi improvisés successivement en nombre indéfini, à la grande joie des auditeurs qui ne se lassent pas, comme nous le ferions, de ce bercement monotone. Il suffit parfois de varier un seul mot, d'ajouter ou de retrancher une négation, pour avoir un nouveau récitatif parallèle.

Les récitatifs peuvent aussi n'être parallèles que deux par deux: chaque couple, formé d'un récitatif 1 et d'un récitatif 2, est séparé du couple suivant par une sorte de récitatif indépendant, sans parallèle, et que nous avons appelé à cause de cela récitatif 0 . Un récitatif 0 commence parfois la série et un autre récitatif 0 la peut terminer, selon I'ordonnance suivante:

$$
R^{0} \cdot R^{1} R^{2} \cdot R^{0} \cdot R^{1} R^{2} \cdot R^{0}
$$

Si nous nous contentons de recueillir, au hasard, une ou deux improvisations dans chaque milieu ethnique de style oral, nous ne pourrons nous empêcher de nous poser ce déroutant problème: Comment des hommes, des femmes, des jeunes filles, presque des enfants, peuvent-ils, à l'improviste, composer oralement des formules rythmiques si gracieuses, si parfaites et si pleines qu'elles forcent même notre admiration, à nous autres raffinés?

Mais si nous continuons, psychologiquement, et méthodiquement, notre enquête dans le même milieu ethnique, le mystère s'éclaircit sans que, pour cela, diminue notre admiration.

En effet, au fur et à mesure que se succèdent devant nous les improvisateurs, nous réentendons, une par une, mais dans des contextes variés, les formules jaillies des lèvres des improvisateurs précédents. 
C'est, pour ainsi dire, comme un jeu merveilleux de dominos vivants: les pièces du jeu restent toujours sensiblement les mêmes [11] avec leurs mêmes attirances réciproques, mais les combinaisons en sont quasi indéfiniment renouvelées.

En poussant encore plus loin les recherches psychologiques, on s'aperçoit que, dans un même milieu ethnique, il peut $y$ avoir plusieurs genres littéraires de style oral : histoire, législation, cosmogonie, morale, philosophie, théologie, médecine, etc., bref toute la science orale de ce milieu ethnique. Cette science n'est évidemment pas aussi avancée que la nôtre et ne s'exprime pas avec notre algébrique terminologie à nous. Mais, au point de vue des individus de ce milieu ethnique, c'est de la science et non pas comme nous le disons trop souvent, de la poésie.

Parfois, il y a même certains genres considérés comme si savants et si techniques qu'ils sont exclusivement réservés aux esprits les mieux doués et les mieux entraînés.

Chacun de ces genres scientifiques a ses clichés plus ou moins spécialisés et aussi ses virtuoses improvisateurs qui peuvent, comme partout, atteindre jusqu'au génie ${ }^{20}$.

Il va sans dire que ces attirances préfixées, ces imbrications verbales coutumières, ces parallélismes clichés, ces récitatifs balancés et comptés, facilitent singulièrement et spontanément la mémorisation de longues séries improvisées ${ }^{21}$.

20 J. DARMESTETER, Chants populaires des Afghans (Paris, 1888-1890), p. CCXI ; M. LONGWORTH DAMES, Popular Poetry of the Baloches (Londres, 1907), pp. XVIIXVIII.

21 Van BIERVLIET, la Mémoire (Paris, 1902), p. 166 ; CLAPARÈDE, I'Association des idées (Paris, 1903), p. 110. 
Mais l'homme a été, toujours et partout, un être trop intelligent et trop industrieux pour ne pas reconnaître, exploiter et développer au maximum les ressources que cette merveilleuse spontanéité de sa nature mettait à sa disposition.

Croyons-le bien: on n'a pas attendu tous les tests savants de nos modernes laboratoires de psychologie expérimentale pour s'apercevoir, que les rythmes, les mélodies, les associations de mots et de sons facilitent étonnamment la mémorisation, la conservation et le rappel des ensembles verbaux. On s'en est d'autant plus préoccupé qu'on avait moins facilement accès à un Larousse de poche ou, à une grande encyclopédie ${ }^{22}$.

Il faudra des volumes -- presque une nouvelle encyclopédie -- pour analyser les merveilleuses, techniques que l'homme a su construire, en dehors de l'écriture, pour tirer de sa mémoire [12] verbale le maximum de rendement. Cette puissance mnémonique est telle que nous autres amnésiques du livre, serions prêts à la nier si les faits vivants, mieux étudiés de jour en jour par la psychologie ethnique, ne nous ramenaient à une humilité plus avertie. Contentons-nous de citer un exemple expérimental pris entre mille.

Chez les Slaves méridionaux, les guslars sont des récitateurs ambulants. Ils sont illettrés, comme c'est le cas d'à peu près tous les improvisateurs et récitateurs des milieux de style oral. Mais là, évidemment, illettré ne veut pas dire ignorant, il s'en faut de beaucoup.

L'opinion populaire doue ces guslars d'une mémoire à première vue surprenante: on vous en cite qui savent $30.000,70.000$ et même plus de 100.000 schèmes rythmiques. Or, le peuple dit vrai.

En soi, le phénomène est aisément explicable, après tout ce que nous avons dit. Les récitations des guslars sont, elles aussi, une juxtaposition de clichés, relativement peu nombreux. Le développement de chacun de ces clichés se fait automatiquement, suivant des règles fixes. Seul leur ordre peut varier. Un bon guslar est celui qui joue de ses clichés comme

22 JULLIOT, l'Éducation de la Mémoire (Paris, 1919), p. 33. 
nous avec des cartes, qui les ordonne diversement suivant le parti qu'il en veut tirer.

Chaque guslar, d'ailleurs, a son genre qui lui est personnel celui-ci se spécialise dans I'histoire de Marko, tel autre célèbre les haïduques.

En outre, chacun se fait une sorte de catalogue oral et mnémotechnique: il range les clichés qui constituent sa «provision en manière de litanie ou de chapelet didactique, comprenant les récitatifs de début des diverses récitations.

L'un des guslars étudiés expérimentalement, un nommé Milovan, et dont la mémoire n'était qu'ordinaire, pouvait réciter ainsi 40.000 schèmes rythmiques à la file.

Instructive enfin, pour la tradition des enseignements de toute nature donnés en style oral, est la constatation suivante: le 18 mars 1885, l'expérimentateur Fr. S. Krauss se fit dicter par un autre guslar, en présence de Milovan, une récitation de 458 schèmes rythmiques que Milovan répéta mot pour mot le 4 octobre 1885, soit sept mois et demi après. Puis Krauss lui fit répéter et nota de nouveau cette même récitation neuf mois plus tard: les variantes furent insignifiantes ${ }^{23}$.

D'ailleurs, quand la conservation impeccable des récitations traditionnelles et surtout religieuses importe à un milieu de style [13] oral, on sait fort bien comment obvier radicalement à ces variantes, même insignifiantes. Donnons-en rapidement un exemple.

Les travaux de M. Rattray, anthropologiste du gouvernement britannique en mission sur la côte occidentale d'Afrique dans le pays des Achantis, viennent d'apporter, avec le fruit de seize années d'études, une

23 Van GENNEP, la Question d'Homère (Paris, 1919), pp. 51-52 ; F.-S. KRAUSS, Slavische Volkforschungen (Leipzig, 1908), pp. 183-189. 
curieuse contribution non seulement à l'étude des langues et à la science musicale, mais aussi à ce chapitre spécial de la psychologie de la récitation que nous esquissons en ce moment.

Nous laisserons donc de côté tout ce qui concerne la linguistique, nous dirons seulement que M. Rattray possède à fond la langue des Achantis et qu'il est parvenu à l'écrire, ce que les indigènes eux-mêmes sont incapables de faire. M. Rattray a pu entendre, écrire et traduire les récitations historiques parce que les Achantis le considéraient comme l'un d'entre eux et comme un homme pieux auquel tout peut être dit et montré.

L'écriture leur étant totalement inconnue, les Achantis, pour conserver leur histoire, ont recours à la seule tradition orale.

Il existe chez eux une caste d'historiens de profession qui racontent les hauts faits des rois en schèmes rythmiques. Ils psalmodient ces récitations sur des mélodies spéciales qui varient avec chaque règne. Leur but rappelle en somme celui des récitateurs de tous les milieux de style oral.

Chaque récitateur a un certain nombre de disciples auxquels il enseigne sa récitation, mot pour mot, et la mélodie appropriée, note pour note. Tout danger de mutilation ou de corruption est évité par ce fait que le récitateur, une fois admis dans la caste, est puni de mort à la moindre faute soit dans le texte, soit dans la psalmodie.

Le résultat de ce système est que les récitations composées depuis plus de huit cents ans nous sont parvenues intactes.

Les langues sont soumises, au cours des siècles, à d'inévitables variations, et celles en particulier qui ne possèdent ni grammaire ni littérature écrites évoluent, en général, plus rapidement que les autres. Le langage achanti ne fait point exception à cette règle et il se trouve que les mots des plus anciennes récitations historiques sont parfaitement incompréhensibles pour la génération actuelle. Seuls les récitateurs en démêlent le sens et sont capables de les traduire en achanti moderne. 
Le chant des récitations historiques est une fonction sacrée, réservée aux seuls récitateurs. M. Rattray dut obtenir du roi une [14] permission spéciale pour qu'il lui fût possible d'enregistrer par le phonographe les précieuses compositions rythmiques des récitateurs achantis ${ }^{24}$.

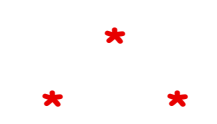

Après de pareilles observations ethniques, on comprend pourquoi nous ne pouvons absolument pas donner à ces récitations de style oral rythmique et à leurs éléments constitutifs le nom de poésie, de strophes, de vers, à cause du sens que, bon gré mal gré, ces mots ont actuellement parmi nous.

Ce serait, en effet, commettre un contresens psychologique énorme et redoutable dans ses conséquences, contresens analogue à celui que l'on commettrait en appelant idoles les statues de nos églises catholiques.

Sans doute, les matériaux physiques et leurs formes extérieures sont à peu près les mêmes. Mais l'attitude mentale des hommes en face de ces matériaux est totalement différente et même opposée.

$\mathrm{Si}$ la science historique, philosophique, théologique, etc., des récitations de style oral est exprimée en formules concrètes, c'est que les langues de ces milieux ethniques n'ont que des expressions concrètes.

Si nous y trouvons des récitatifs faits de schèmes rythmiques, c'est que toutes ces récitations doivent être scrupuleusement retenues par coeur et que les rythmes, les mélodies, etc., sont des adjuvants considérables de la mémorisation.

Chez nous, au contraire, nous avons proclamé doctement que «savoir par coeur n'est pas savoir», sans peut-être nous douter que nous

24 PFISTER, les Chansons historiques des Achantis. Revue musicale, avril 1923, pp. 230-284 : -- RATTRAY, Ashanti(Oxford, 1923), p. 219. 
condamnions ainsi la science réelle de $99 \%$, des hommes qui ont peuplé et peuplent encore la planète.

Notre didactique, à tort ou à raison ${ }^{25}$, a délaissé de siècle en siècle les procédés spontanés du style oral qui a fait place à notre style écrit. L'antique style oral, didactique et vivant, est passé du domaine utilitaire dans le domaine purement esthétique, loin de la foule.

Là, il est devenu cette préparation livresque, artificielle, splendidement isolée, que nous nommons poésie et même poésie pure ${ }^{26}$. Les adjuvants didactiques les plus saillants (allitération, rime, etc.), ont changé complètement de nature et se' sont mués en obstacles [15] de plus en plus difficiles, rivalisant parfois avec le casse-tête des «mots croisés ».

Aujourd'hui, il ne viendra à l'idée d'aucun de nos professeurs de philosophie, de théologie, d'histoire, d'astronomie, de donner son enseignement sous forme de sonnets parallèles.

Or, ce simple fait montre, expérimentalement, pour ainsi dire, l'abîme psychologique et social qui existe entre le style oral et la poésie.

Curieuse métamorphose d'une institution humaine et qui doit attirer l'attention du psychologue et de l'ethnographe: ce qui était, par nature, essentiellement didactique et oral est devenu, chez nous, essentiellement esthétique et écrit.

Parfois cependant, en plein milieu de style oral, les récitations composées oralement et sues par cœur peuvent, tôt ou tard, être mises par écrit sur des stèles, sur des briques, sur des peaux de bête, etc.

\footnotetext{
25 À tort, semble-t-il, si l'on en juge d'après les intéressantes innovations de la pédagogie moderne. Voir, là-dessus MULOT, Système Éducatif français (Paris, 1921).

26 Henri BREMOND, la Poésie pure (Paris, 1926).
} 
Mais n'allons pas confondre ces graphies avec nos livres actuels que nous parcourons d'un œil rapide. Elles sont, pour ainsi dire, des «textesétalons», des «témoignages» qui servent à confirmer ou à rectifier la teneur traditionnelle des récitations vivantes. Aussi ces «textestémoins » sont-ils souvent conservés religieusement dans les temples, sous la garde sévère d'une caste sacerdotale.

Dans ces textes oraux « mis par écrit » et essentiellement faits pour être sus par cœur, nous trouverons donc encore, naturellement, des récitations rythmiques, mais ce ne seront pas des poèmes.

Nous y trouverons des récitatifs didactiques, mais ce ne seront pas des strophes.

Nous y rencontrerons de fréquentes répétitions mnémotechniques, mais il faudra bien nous garder de les prendre pour des « dittographies» ou pour des «gloses de seconds rédacteurs maladroits». En effet, les récitations vivantes, et qui n'ont jamais été «mises par écrit», présentent, à chaque instant, des répétitions analogues et destinées au même but didactique.

Lorsque les milieux ethniques vivants nous ont familiarisés avec les procédés psycho-physiologiques et universels du style oral, l'idée nous vient tout naturellement d'utiliser nos découvertes expérimentales pour analyser la structure stylistique des textes que les milieux ethniques de jadis nous ont laissés, milieux ethniques que I'histoire nous désigne comme ayant été de style oral tout en ayant parfois connu parfaitement l'écriture. (C'est ce que [16] César, par exemple, nous dit des druides dans ses Commentaires, VI, 14. Sur les Rabbis dIsraël ${ }^{27}$, voir Towa Perlow, l'Éducation et l'Enseignement chez les Juifs à l'époque talmudique, Paris, 1931, 1 pp. 37 et 54.)

Au strict point de vue de la psychologie linguistique, tous les milieux ethniques sont, en soi, également intéressants. Une grande civilisation comme celle des Incas de l'ancien Pérou, avec ses récitations

27 Marcel JOUSSE, études sur la Psychologie du Geste. Les Rabbis d'Israël : les Récitatifs rythmiques parallèles. T. I, genre de la Maxime, Paris, 1930. 
traditionnelles, ne peut nous laisser indifférents. Ainsi en est-il des anciens Chinois, des Arabes préislamiques, etc.

Mais à cause de notre formation classique et quasi exclusivement gréco-latine, le milieu homérique nous touche de plus près. Or, après avoir pratiqué Homère pendant un certain nombre d'années, on arrive à reconnaître, une par une, les formules de style oral familières à son milieu ethnique. C'est avec ces mosaíques d'expressions, toutes faites et à l'usage de tous les (mot grec), que le grand rythmeur aveugle a cependant «prononcé »-- nous ne disons pas : « écrit»-- deux des plus purs chefsd'œuvre qui soient sortis des lèvres humaines.

Sur cette découverte, au premier abord déconcertante, d'un Homère fait de quelques centaines de formules ethniques et impersonnelles, nous avons été particulièrement heureux de nous rencontrer avec notre grand linguiste français, M. le professeur Meillet ${ }^{28}$.

Une fois l'attention attirée sur ce point, le fait est si frappant qu'on peut arriver à retrouver, sans faute, le grec même d'Homère, en se servant d'une traduction latine, par exemple, pourvu évidemment qu'elle soit très fidèlement, très littéralement « décalquée». (Telle est la traduction Firmin-Didot.) Il faut d'abord apprendre par cœur toutes les formules grecques des (mot grec) et voir comment notre traduction latine les décalque (parfois avec des décalquec synonymes). C'est alors un exercice intellectuel passionnant de s'essayer à « reconstruire » Homère en remplaçant les expressions latines décalques par les quelques centaines de formules grecques correspondantes.

Donnons-en quelques exemples, pris au hasard. Soit un binaire grec formulaire avec son décalque latin:

28 A. MEILLET : les origines indo-européennes des mètres grecs, Paris, 1923, p. 61. 

(en grec)
(en grec)
Ut dicam quae me animus
In pectoribus jubet $H 369$.

Ce sera un jeu aisé de remplacer par de l'Homère, absolument [17] impeccable, le pointillé que nous traçons au-dessus de chacun des décalques suivants:

Ut dicam quae me animus

Ut dicam quae me animus …….....................

Ut dicam quae me animus

Soit une nouvelle formule:

(en grec)

Sic dixit : neque inobsequens erat $H 43$.

Au-dessus des décalques latins, identiques ou synonymes, la formule grecque originale viendra automatiquement et conforme à Homère:

Sic dixit ; nec non paruit B 166.

Sic dixit ; nec non obsecutus est epsilon 43. 
Il en sera de même pour cette autre formule ethnique grecque qui nous est familière, à nous aussi :
(en grec)
(en grec)
dea cacsiis oculis
Minerva A 206.

et dont nous rencontrons les décalques latins à chaque instant:

\begin{tabular}{|c|c|}
\hline dea caesiis oculis & Minerva \\
\hline & $\cdots$ \\
\hline & Minerva \\
\hline
\end{tabular}

Ces deux dernières formules nous permettent même, dès maintenant, de saisir sur le vif et de rejouer en nous le mécanisme psychophysiologique des «enchaînements » oraux tel qu'il se jouait dans les organes laryngo-buccaux du grand rythmeur grec. En effet devant le décalque latin suivant:

Sic dixit : nec non piruit

dea caesiis oculis Minerva

B 166.

nous constatons immédiatement qu'il n'est que l' « enchaînement 》 de deux formules déjà connues et nous pouvons ainsi reconstruire, sans faute, l'en semble homérique original :

$$
\text { (en grec) (engrec) }
$$

Les nombreuses questions que soulève cette méthodologie du [18] style oral appliquée aux compositions homériques ont été longuement 
étudiées par M. Milman Parry ${ }^{29}$. Nous nous permettons de conseiller aux ethnographes la lecture des ouvrages si suggestifs d'un des disciples de M. Meillet. La psychologie ethnique et la linguistique philologique pourraient-elles collaborer sous plus favorables auspices?

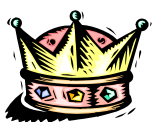

29 Milman PARRY: Studies in the Epic Technique of Oral Verse-Making. I. Homer and Homeric Style (Harvard Studies in Classical Philology,vol. XLI, 1930, pp.73-147). On lira avec avantage cette synthèse avant les deux ouvrages parus précédemment: Milman PARRY : l'Épithète traditionnelle dans Homère, Paris, 1928 ; les Formules et la Métrique d'Homère, Paris, 1928. 